\title{
Modeling the Separating Pedestrian Flow in T-Shaped Passage Based on Guide Sign
}

\author{
Hong-fei Jia, ${ }^{1}$ Yong-xing Li, ${ }^{1}$ Li-li Yang, ${ }^{1,2}$ and Ya-nan Zhou ${ }^{1}$ \\ ${ }^{1}$ School of Transportation, Jilin University, Changchun, Jilin 130025, China \\ ${ }^{2}$ School of Management, Jilin University, Changchun, Jilin 130025, China \\ Correspondence should be addressed to Li-li Yang; yangll105@126.com
}

Received 4 June 2016; Accepted 11 July 2016

Academic Editor: Chris Goodrich

Copyright (c) 2016 Hong-fei Jia et al. This is an open access article distributed under the Creative Commons Attribution License, which permits unrestricted use, distribution, and reproduction in any medium, provided the original work is properly cited.

Considering the actual situation of separating pedestrian flow in T-shaped passage, the guide sign is set to guide the pedestrians and subconscious strength is introduced to show the effect of guide sign. Pedestrian subconscious strength model is established, and the subconscious strength calculation result is added to the pedestrian simulation model which is based on cellular automata. On the platform of MATLAB software, separating pedestrian flow simulation with the effect of guide sign is realized. Simulations indicate that, compared with the separating pedestrian flow without guide sign, the efficiency of pedestrians passing with guide sign is higher. Analyzing the effect of guide sign in different positions, the suitable position of guide sign is obtained.

\section{Introduction}

T-shaped passage is the common pedestrian facilities in the hub. With the different walking directions, pedestrians would wave in T-shaped passage, and the crowing phenomenon may happen in some time [1]. In T-shaped passage, separating pedestrian flow and merging pedestrian flow are the familiar waving behaviors. Researching the waving phenomenon in Tshaped passage and taking some effective measures to reduce pedestrian conflicts have important practical significance.

At present, many studies have been made on pedestrian flow at different cases, for example, pedestrian flow simulation in bidirectional passage [2-4], pedestrians evacuation on stairs [5], pedestrian evacuation with panic [6], pedestrians evacuation in rooms with internal obstacles and multiple exits [7], and pedestrian flow analysis and simulation in T-shaped passage [8-13]. In T-shaped passage, Boltes et al. [8] and Zhang et al. [9] analyze the pedestrian flow dynamics with empirical data, respectively, and the pedestrian flow fundamental diagram is obtained. Tajima and Nagatani simulate the merging pedestrian flow by using the lattice gas model of biased random walkers [10]. Chen et al. establish a forcedriving cellular automata model to investigate the evacuation behaviors of pedestrians at a T-shaped intersection, and the evacuation behaviors of pedestrians are simulated in terms of different pedestrian density [11]. Peng and Chou study the conformation of pedestrian congestion in a " $\mathrm{T}$ " intersection by using multifloor fields cellular automata [12]. $\mathrm{Fu}$ et al. simulate the interaction of separating pedestrian flow interlaced in a T-shaped passage with a modified multifield cellular automaton updating synchronously. And in the simulation, they find how the entrance density and the pedestrians expecting to move to the left exit affect the phase transition diagram of pedestrian flow interlacing and separating [1].

For improving the efficiency of pedestrians passing, setting the guide sign is the usually used method. Xiong et al. study the impact of traffic sign on pedestrian walking behavior, and the one-way pedestrian flow has been simulated based on cellular automata [13]. Song et al. analyze the effects of direction sign in pedestrian evacuation process and build the pedestrian evacuation model [14]. With the research and the actual observation, we can know that the efficiency of pedestrians passing is not high because of the conflicts in the separating process of pedestrians. Setting the guide sign in front of the separating of the pedestrians can reduce the pedestrian conflicts in the separating process of pedestrians [13-15]. 


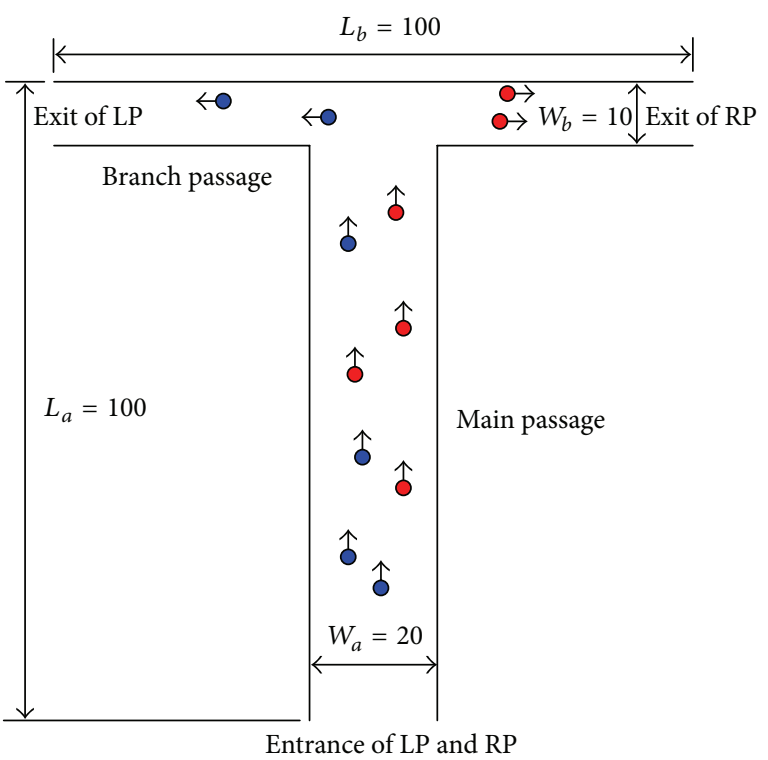

FIgURE 1: Simulation scenario of T-shaped passage.

In this paper, we transfer the effect of guide sign into the subconscious strength and add the subconscious strength to the pedestrian simulation model which is based on cellular automata, and in this way, the effect of guide sign in separating pedestrian flow is simulated.

\section{Simulation Model of Separating Pedestrian Flow Based on Guide Sign}

2.1. Assumption Conditions. There are four assumptions that have been made in the separating pedestrian flow simulation.

Assumption 1. All the pedestrians have the same expectation speed.

Assumption 2. It is without considering pedestrian individual difference.

Assumption 3. The sight distance of the pedestrian is $6 \mathrm{~m}$, and all the pedestrians are the same.

Assumption 4. All the pedestrians are influenced by guide sign; that is to say, all pedestrians have the subconscious behavior to walk along the left-hand or right-hand side with the effect of guide sign.

2.2. Simulation Scenario. Assume a T-shaped passage which is composed of main passage and branch passage shown in Figure 1. The lengths of main passage and branch passage are all 100 cells. The width of main passage is 20 cells, and the width of branch passage is 10 cells. The size of each cell is $0.4 \mathrm{~m} \times 0.4 \mathrm{~m}$, which is the typical space occupied by a pedestrian $[16,17]$. Only one pedestrian can be contained in a cell at most. Pedestrians generated at the down origin of main passage and who walk away at the left side exit of branch passage are set as LP. Pedestrians generated at the origin of main passage and who walk away at the right side exit of branch passage are set as RP. Pedestrians move one cell each time-step.

2.3. Floor Field. The floor field has been proposed many years ago, and it has been widely used in pedestrian flow simulation [18]. According to the relevant research $[1,18]$, in the calculation process of static floor field, we can think that the target exit and nearest boundary are the main factors. The calculation formula is as follows:

$$
S_{i j}=a \cdot S_{i j}^{\prime}+b \cdot S_{i j}^{\prime \prime},
$$

where $S_{i j}$ is the value of static floor field at cell $(i, j) ; S_{i j}^{\prime}$ is the value of static floor field at cell $(i, j)$ formed by the exit; $S_{i j}^{\prime \prime}$ is the value of static floor field at cell $(i, j)$ formed by the nearest boundary; $a$ and $b$ are the coefficients.

Pedestrians move from cell $(i, j)$ to destination in horizontal, vertical, and diagonal directions. The value of static floor field at cell $(i, j)$ formed by the exit can be calculated as follows:

$$
S_{i j}^{\prime}=\left(\max _{i, j} d_{i j}\right)-d_{i j}
$$

where $d_{i j}$ is the distance from cell $(i, j)$ to the exit.

Considering the phenomenon that pedestrians tend to keep a certain distance from the boundary [1], the value of static floor field at cell $(i, j)$ formed by the nearest boundary can be calculated as follows:

$$
S_{i j}^{\prime \prime}=d_{i j}^{\prime}
$$

where $d_{i j}^{\prime}$ is the distance from cell $(i, j)$ to the nearest boundary.

Only with the static floor field, the problem of "dead end" may appear, meaning that pedestrians moving in opposite directions will get stuck into the same cell, even though there are other cells available nearby. In the walking process, the pedestrian would try to avoid the densely populated area for comfortable walking. Considering this, the dynamic floor field is introduced as follows:

$$
D_{i j}=1-\frac{r_{i j}}{N_{i j}},
$$

where $D_{i j}$ is the value of dynamic floor field at cell $(i, j) ; r_{i j}$ is the number of cell $(i, j)$ and its neighbors which have been occupied by pedestrians; $N_{i j}$ is the number of cell $(i, j)$ and its neighbors.

2.4. Addition of the Guide Sign. The guide sign is set to reduce the pedestrian conflicts, and the position of guide sign is shown in Figure 2. The length of the guiding zone is $L_{m}$ and the length of pedestrian sight distance is $L_{s}$. According to related research [19], the length of pedestrian sight distance $L_{s}$ is equal to 15 cells $(6 \mathrm{~m})$. The yellow zone shown in Figure 2 is the guide zone with the length $L_{m}+L_{s}$ and width $W_{a}$.

When pedestrians arrive at the guide zone, under the effect of guide sign, LP (RP) generate the subconscious 


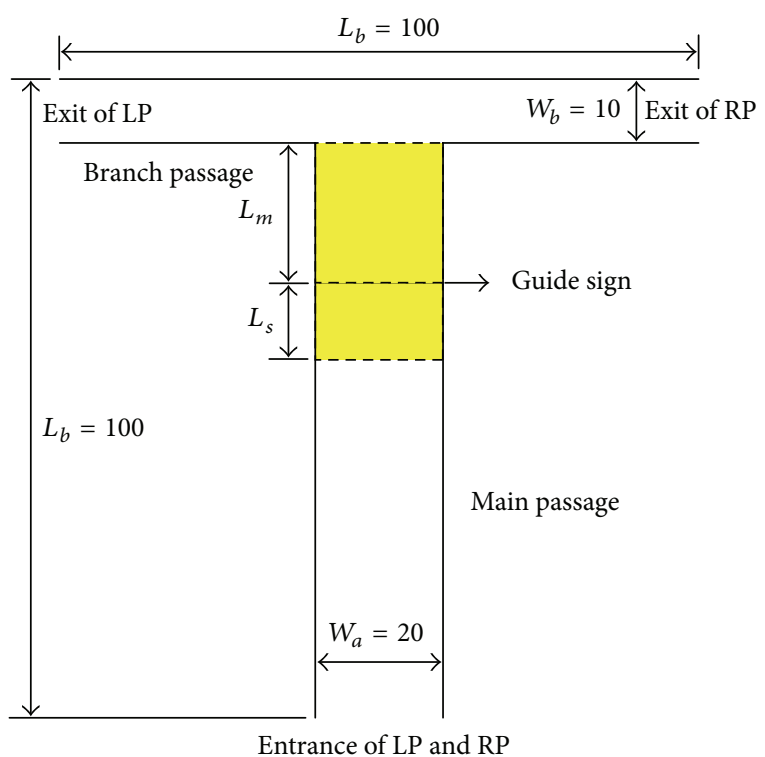

FIgURE 2: Schematic diagram of the position of guide sign.

behavior to walk along the left-hand (right-hand) side. The subconscious strength $M$ is introduced to show the effect of guide sign. The subconscious strength $M$ is calculated as follows:

$$
M=\alpha \cdot M_{1} \cdot M_{2},
$$

where $M$ is subconscious strength of LP (RP); $M_{1}$ is the subconscious strength formed by the horizontal position of LP (RP); $M_{2}$ is the subconscious strength formed by the vertical position of LP (RP); $\alpha$ is the undetermined coefficient, and $0<\alpha \leq 1$.

In the guide zone, when the distance between the LP (RP) and left (right) boundary of main passage is larger, the subconscious of LP (RP) to walk along left-hand (right-hand) side is stronger; namely, the value of $M_{1}$ is bigger; on the contrary, the value of $M_{1}$ is smaller.

The subconscious strength $M_{1}$ for LP is calculated as follows:

$$
M_{1}=1-\frac{1}{1+\exp ((R-0.4) / 0.08)}, \quad R=\frac{W_{a}-d_{1}}{W_{a}} .
$$
follows:

The subconscious strength $M_{1}$ for RP is calculated as

$$
M_{1}=1-\frac{1}{1+\exp ((R-0.4) / 0.08)}, \quad R=\frac{d_{1}}{W_{a}},
$$

where $d_{1}$ is the distance between pedestrian and right boundary of main passage, and $0<d_{1}<W_{a}$; $W_{a}$ is the width of main passage; $R$ is the ratio of distance between LP (RP) and left (right) boundary and the width of main passage, and $0<R<1$.

The distance between LP (RP) and down boundary of branch passage is also the main influence factor for subconscious strength. When the distance between LP (RP) and down boundary of branch passage is larger, the subconscious of LP (RP) to walk along left-hand (right-hand) side is smaller; namely, the value of $M_{2}$ is smaller; on the contrary, the value of $M_{2}$ is bigger. The subconscious strength $M_{2}$ of LP (RP) is calculated as follows:

$$
M_{2}=\exp \left(-\frac{d_{2}}{L_{m}+L_{s}}\right)
$$

where $d_{2}$ is the distance between pedestrian and the down boundary of branch passage.

When we assume that $L_{m}=2 \mathrm{~m}$ and $L_{s}=6 \mathrm{~m}$, we can draw the curve of the subconscious strength $M$ for LP shown in Figure 3 and the curve of the subconscious strength $M$ for RP shown in Figure 4. From Figures 3 and 4, we can clearly know the changing tendency of the subconscious strength, and it is consistent with the actual situation.

2.5. Transition Probability. Cellular automata Von Neumann motion rule $[20,21]$ is used in this paper. In Figure 5, including the position occupied by himself or herself, there are five positions for pedestrian to go. Transition probability of pedestrian to each position is shown in Figure 6.

With the cellular automata Von Neumann motion rule, the transition probability of pedestrian without guide sign is calculated as follows:

$$
\begin{aligned}
& P_{i j}=\frac{1}{N} \cdot \exp \left(k_{S} \cdot S_{i j}\right) \cdot \exp \left(k_{D} \cdot D_{i j}\right) \cdot\left(1-\mu_{i j}\right) \cdot \xi_{i j}, \\
& N=\sum_{i, j} \exp \left(k_{S} \cdot S_{i j}\right) \cdot \exp \left(k_{D} \cdot D_{i j}\right) \cdot\left(1-\mu_{i j}\right) \cdot \xi_{i j} .
\end{aligned}
$$

The transition probability of pedestrian with guide sign is calculated as follows:

$P_{i j}$

$$
= \begin{cases}\frac{1 / N \cdot\left[\exp \left(k_{S} \cdot S_{i j}\right) \cdot \exp \left(k_{D} \cdot D_{i j}\right) \cdot\left(1-\mu_{i j}\right) \cdot \xi_{i j}\right]+M}{1+M}, & \text { left-hand direction for LP (right-hand direction for RP) } \\ \frac{1 / N \cdot \exp \left(k_{S} \cdot S_{i j}\right) \cdot \exp \left(k_{D} \cdot D_{i j}\right) \cdot\left(1-\mu_{i j}\right) \cdot \xi_{i j}}{1+M}, & \text { otherwise, }\end{cases}
$$




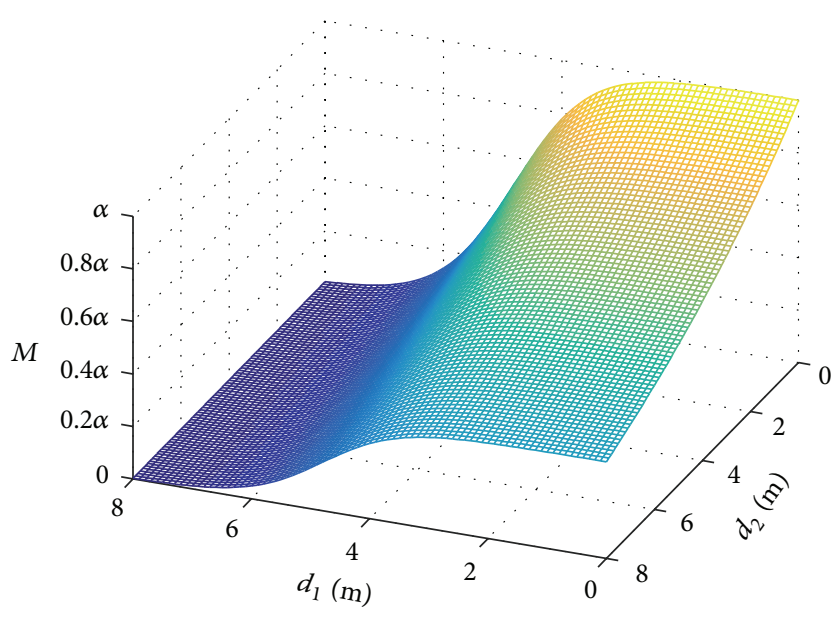

FIgURE 3: The curve of the subconscious strength $M$ for LP.

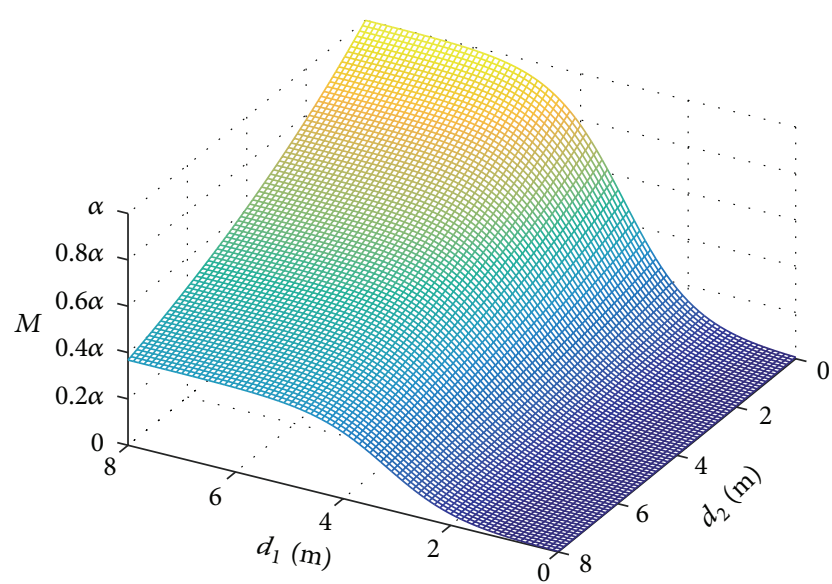

FIgURE 4: The curve of the subconscious strength $M$ for RP.

where $N$ is the normalized parameter; $k_{S}$ is the coefficient of static floor field, which reflects the degree of pedestrians' familiarity to the inherent characteristics of the scenario; $k_{D}$ is the coefficient of dynamic floor field, which reflects the following trend of pedestrian in procession; $\mu_{i j}$ is the parameter which judges whether the cell is occupied by pedestrian or not, and $\mu_{i j}=1$ if cell $(i, j)$ is occupied and $\mu_{i j}=0$ otherwise; $\xi_{i j}$ is the parameter which judges whether the cell is occupied by wall or barrier or not, and $\xi_{i j}=0$ if cell $(i, j)$ is occupied and $\xi_{i j}=1$ otherwise.

According to relevant research [1] and many simulation experiments, in order to be closer to reality, we propose that $k_{S}=1, k_{D}=0.4, a=1, b=0.4$, and $\alpha=0.6$. With the platform of MATLAB software, the separating pedestrian flow simulation is realized.

\section{Simulation Results and Discussion}

Separating pedestrian flow simulation is realized on the platform of MATLAB software. Separating pedestrian flow

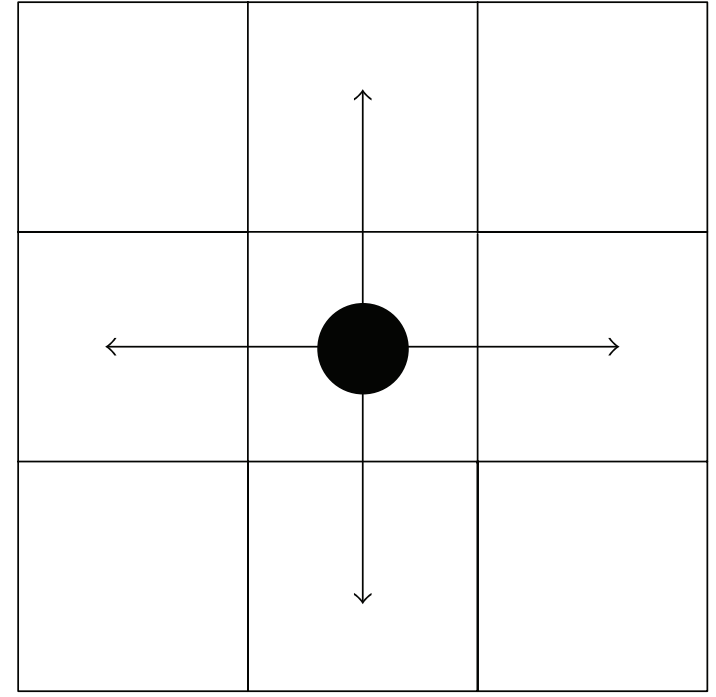

FIgURE 5: Von Neumann motion rule.

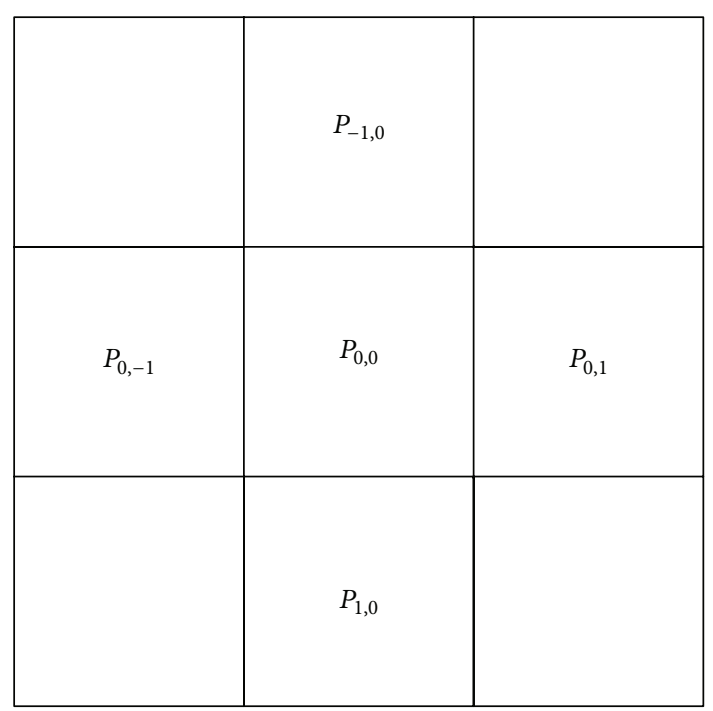

FIgURE 6: Transition probability.

simulation process without and with guide sign is, respectively, shown in Figures 7 and 8. The blue solid circles are the LP and the red solid circles are the RP.

In the simulation, there are three stages without and with guide sign, respectively.

The First Stage. At the down origin of main passage, LP and $\mathrm{RP}$ are generated and walk towards the left side and right side exit of branch passage, respectively.

The Second Stage. Without the effect of guide sign, LP and RP arrive at the waving zone and interweave.

With the effect of guide sign, the pedestrians see the guide sign and generate the subconscious behavior. LP tend to walk along the left-hand side, and RP tend to walk along the righthand side. When pedestrians walk away from the guide zone 


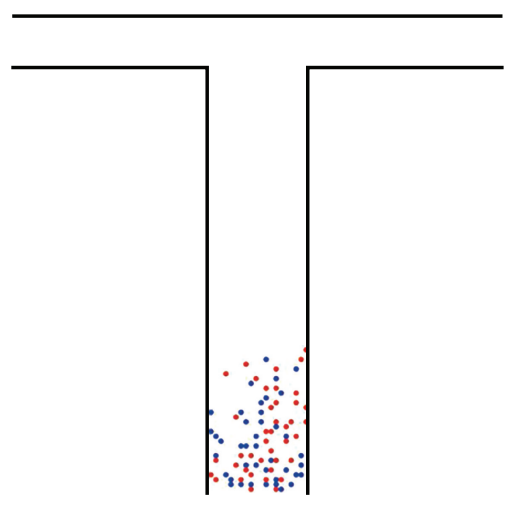

(a)

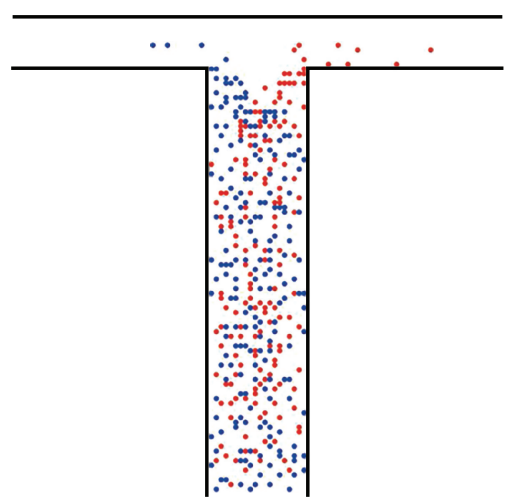

(b)

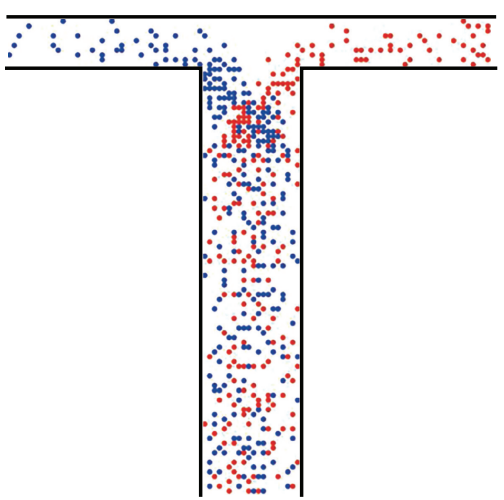

(c)

FIGURE 7: The simulation of separating pedestrian flow without guide sign. (a) LP and RP are generated at the down origin of main passage; (b) LP and RP arrive at the waving zone and interweave; (c) LP and RP arrive at the exit and walk away.

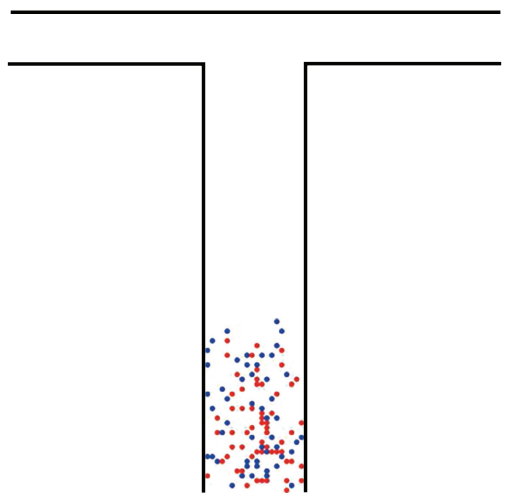

(a)

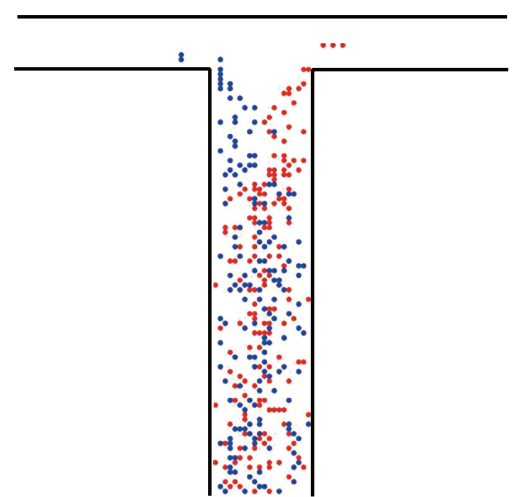

(b)

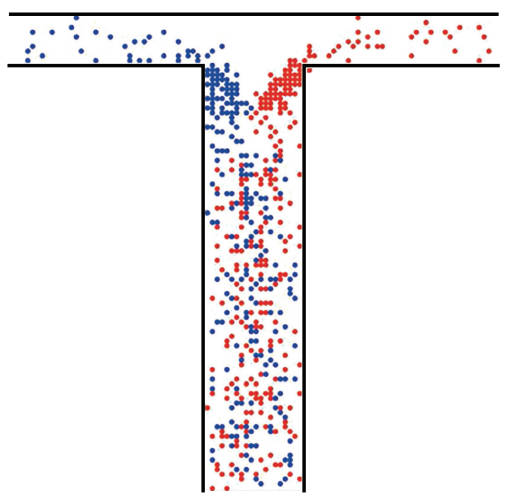

(c)

FIGURE 8: The simulation of separating pedestrian flow with guide sign. (a) LP and RP are generated at the down origin of main passage; (b) LP and RP see the guide sign and generate the subconscious behavior; (c) LP and RP arrive at the exit and walk away.

and arrive at the branch passage, the subconscious behavior disappears.

The Last Stage. LP and RP arrive at the left side and right side exit of branch passage, respectively, and walk away.

In the simulation of separating pedestrian flow without guide sign, the " $\mathrm{X}$ " shaped separating pedestrian flow appears in the waving zone (see Figure $7(\mathrm{c})$ ), and pedestrians crowed at the turning corner. These are all consistent with the actual situation. In the simulation of separating pedestrian flow with guide sign, LP walk along the left-hand and RP walk along the right-hand side, respectively. The weaving behavior is weakened, and pedestrians crowed at the turning corner.

Assuming that the rate of pedestrian generation is constant and taking 50 times independent simulation experiments, when the pedestrian flow is stable, the number of pedestrians passing the passage during 200 time-steps is shown in Figure 9.

From Figure 9, we can know that the number of pedestrians passing the passage with guide sign is bigger than that without guide sign. The mean number of pedestrians

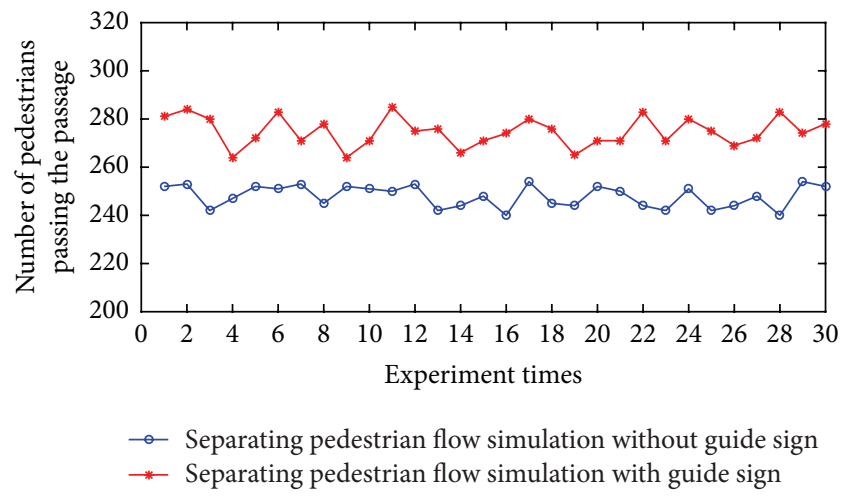

FIgURE 9: The number of pedestrians passing the passage without and with guide sign.

passing the passage without and with guide sign is 248 and 275 , respectively. It indicates that the guide sign is useful for reducing the conflicts and improving the efficiency of pedestrians passing. 


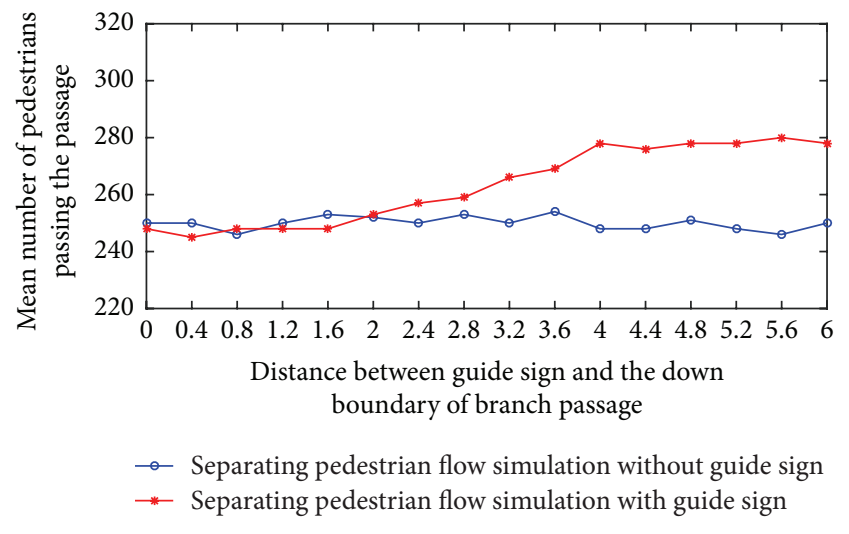

FIGURE 10: The mean number of pedestrians passing the passage with different position of guide sign.

With the constant rate of pedestrian generation, we analyze the influence of the position of guide sign for the efficiency of pedestrians passing. Taking 50 times independent simulation experiments, with different position of guide sign, the change of mean number of pedestrians passing is shown in Figure 10.

In the separating pedestrian flow simulation without and with guide sign, when the distance between guide sign and the down boundary of branch passage is less than $2 \mathrm{~m}$, the difference of the number of pedestrians passing the passage is small. In the separating pedestrian flow simulation with guide sign, with the increasing of the distance between guide sign and the down boundary of branch passage, the conflicts reduce and the number of pedestrians passing the passage increases. When the distance between guide sign and down boundary of branch passage is bigger than $4 \mathrm{~m}$, the difference in the number of pedestrians passing the passage is no longer increasing. It indicates that when we set the guide sign, the distance between guide sign and down boundary of branch passage is suitable for bigger than $4 \mathrm{~m}$.

\section{Conclusion}

(1) With the effect of guide sign, pedestrians generate the subconscious behavior for walking along the lefthand or right-hand side. Subconscious strength is introduced to show the effect of guide sign. Pedestrian subconscious strength is added to the pedestrian simulation model which is based on cellular automata. Separating pedestrian flow simulation with the effect of guide sign is realized.

(2) In the separating pedestrian flow simulation of Tshaped passage, compared with the separating pedestrian flow without guide sign, the efficiency of pedestrians passing the passage is improved with the effect of guide sign.

(3) Through many independent simulation experiments, we analyze the changing of the efficiency of pedestrians passing the passage with the changing position of guide sign, and suitable distance between guide sign and the down boundary of branch passage is obtained.

\section{Competing Interests}

The authors declare that there is no conflict of interests regarding the publication of this paper.

\section{Acknowledgments}

The research is supported by the National Natural Science Foundation of China (Grant nos. 51278221 and 51378076) and the Science Technology Development Project of Jilin Province, China (Grant no. 20140204027SF).

\section{References}

[1] Z. J. Fu, L. Z. Yang, P. Rao, and T. L. Zhang, "Interactions of pedestrians interlaced in T-shaped structure using a modified multi-field cellular automaton," International Journal of Modern Physics C, vol. 24, no. 4, Article ID 1350024, 2013.

[2] H. Yue, H. Guan, J. Zhang, and C. Shao, "Study on bi-direction pedestrian flow using cellular automata simulation," Physica A: Statistical Mechanics and its Applications, vol. 389, no. 3, pp. 527539,2010

[3] J. Ma, W.-G. Song, J. Zhang, S.-M. Lo, and G.-X. Liao, "knearest-neighbor interaction induced self-organized pedestrian counter flow," Physica A: Statistical Mechanics and Its Applications, vol. 389, no. 10, pp. 2101-2117, 2010.

[4] W. Guo, X. L. Wang, and X. P. Zheng, "Lane formation in pedestrian counterflows driven by a potential field considering following and avoidance behaviours," Physica A: Statistical Mechanics and its Applications, vol. 432, pp. 87-101, 2015.

[5] Y. C. Qu, Z. Y. Gao, Y. Xiao, and X. G. Li, "Modeling the pedestrian's movement and simulating evacuation dynamics on stairs," Safety Science, vol. 70, pp. 189-201, 2014.

[6] J. H. Wang, L. Zhang, Q. Y. Shi, P. Yang, and X. M. Hu, "Modeling and simulating for congestion pedestrian evacuation with panic," Physica A: Statistical Mechanics and Its Applications, vol. 428, pp. 396-409, 2015.

[7] H.-J. Huang and R.-Y. Guo, "Static floor field and exit choice for pedestrian evacuation in rooms with internal obstacles and multiple exits," Physical Review E, vol. 78, no. 2, Article ID 021131, 2008.

[8] M. Boltes, J. Zhang, A. Seyfried, and B. Steffen, "Pedestrian dynamics-experiments, analysis, modeling," in Proceedings of the IEEE International Conference on Computer Vision Workshops (ICCV '11), pp. 158-165, Barcelona, Spain, November 2011.

[9] J. Zhang, W. Klingsch, A. Schadschneider, and A. Seyfried, "Transitions in pedestrian fundamental diagrams of straight corridors and T-junctions," Journal of Statistical Mechanics: Theory and Experiment, vol. 2011, no. 6, Article ID P06004, 2011.

[10] Y. Tajima and T. Nagatani, "Clogging transition of pedestrian flow in T-shaped channel," Physica A: Statistical Mechanics and Its Applications, vol. 303, no. 1-2, pp. 239-250, 2002.

[11] C.-K. Chen, J. Li, and D. Zhang, "Study on evacuation behaviors at a T-shaped intersection by a force-driving cellular automata model," Physica A: Statistical Mechanics and its Applications, vol. 391, no. 7, pp. 2408-2420, 2012. 
[12] Y.-C. Peng and C.-I. Chou, "Simulation of pedestrian flow through a ' $\mathrm{T}$ ' intersection: a multi-floor field cellular automata approach," Computer Physics Communications, vol. 182, no. 1, pp. 205-208, 2011.

[13] H. Xiong, P. F. Yao, X. D. Guo, C. L. Chu, and W. H. Wang, "Impact of traffic sign on pedestrians' walking behavior," Mathematical Problems in Engineering, vol. 2015, Article ID 826152, 10 pages, 2015.

[14] B. X. Song, Z. Z. Wu, and Z. H. Xie, "Study on occupant evacuation model considering influence of direction sign," China Safety Science Journal, vol. 21, no. 12, pp. 27-33, 2011.

[15] J. Lin, R. Song, J. Dai, and P. Jiao, "Pedestrian guiding signs optimization for airport terminal," Discrete Dynamics in Nature and Society, vol. 2014, Article ID 125910, 14 pages, 2014.

[16] J. Li, S. Y. Fu, H. B. He, H. F. Jia, Y. Z. Li, and Y. Guo, "Simulating large-scale pedestrian movement using CA and event driven model: methodology and case study," Physica A: Statistical Mechanics and Its Applications, vol. 437, pp. 304-321, 2015.

[17] H. Yue, B.-Y. Zhang, C.-F. Shao, and Y. Xing, "Exit selection strategy in pedestrian evacuation simulation with multi-exits," Chinese Physics B, vol. 23, no. 5, Article ID 050512, 2014.

[18] C. Burstedde, K. Klauck, A. Schadschneider, and J. Zittartz, "Simulation of pedestrian dynamics using a two-dimensional cellular automaton," Physica A: Statistical Mechanics and its Applications, vol. 295, no. 3-4, pp. 507-525, 2001.

[19] Z.-J. Wang, F. Chen, and Z.-H. Shi, "Agent-based realization of social force model and simulation of pedestrians in subway passageway," Journal of South China University of Technology, vol. 41, no. 4, pp. 90-95, 2013.

[20] M. J. Liao and G. Liu, "Modeling passenger behavior in nonpayment areas at rail transit stations," Transportation Research Record: Journal of the Transportation Research Board, vol. 2534, pp. 101-108, 2015.

[21] L.-L. Lu, G. Ren, W. Wang, and Y. Wang, "Modeling walking behavior of pedestrian groups with floor field cellular automaton approach," Chinese Physics B, vol. 23, no. 8, Article ID 088901, 2014. 


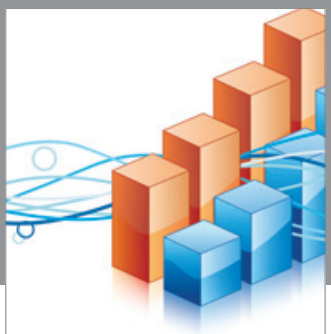

Advances in

Operations Research

vatem alat4

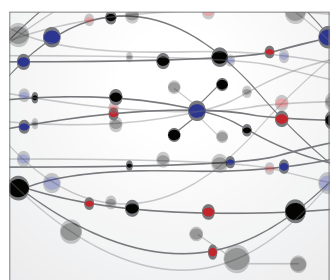

\section{The Scientific} World Journal
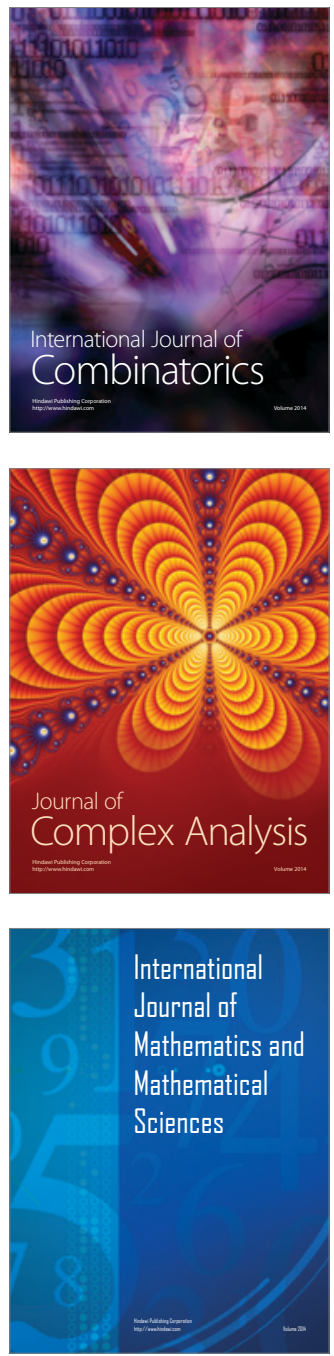
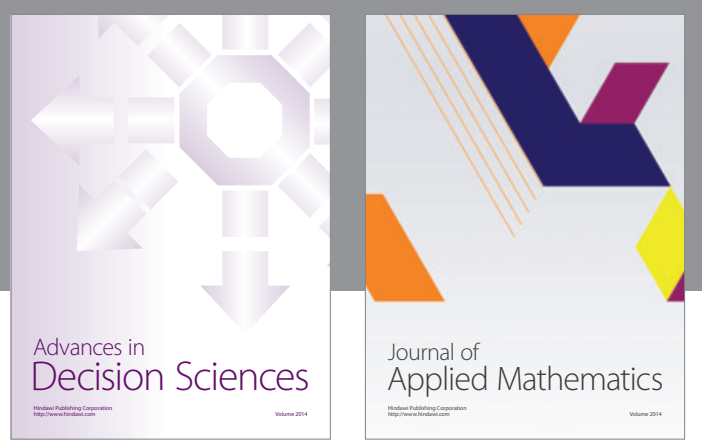

Algebra

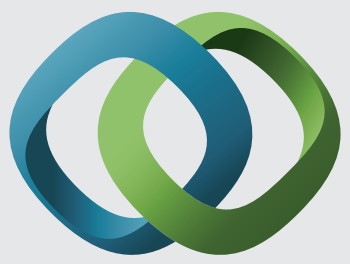

\section{Hindawi}

Submit your manuscripts at

http://www.hindawi.com
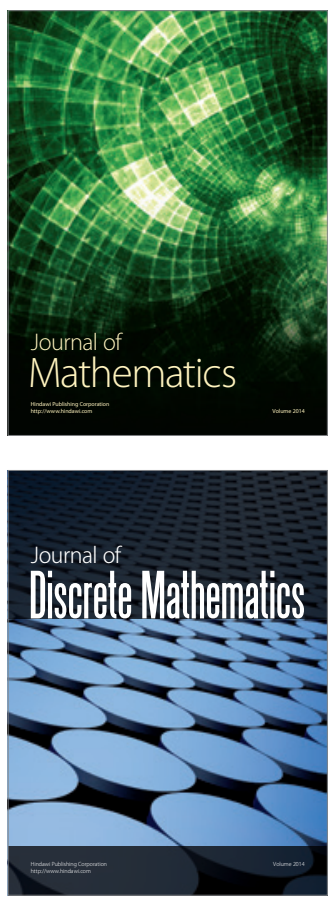

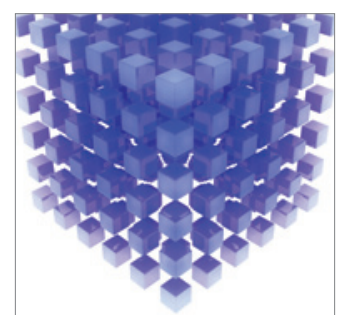

Mathematical Problems in Engineering
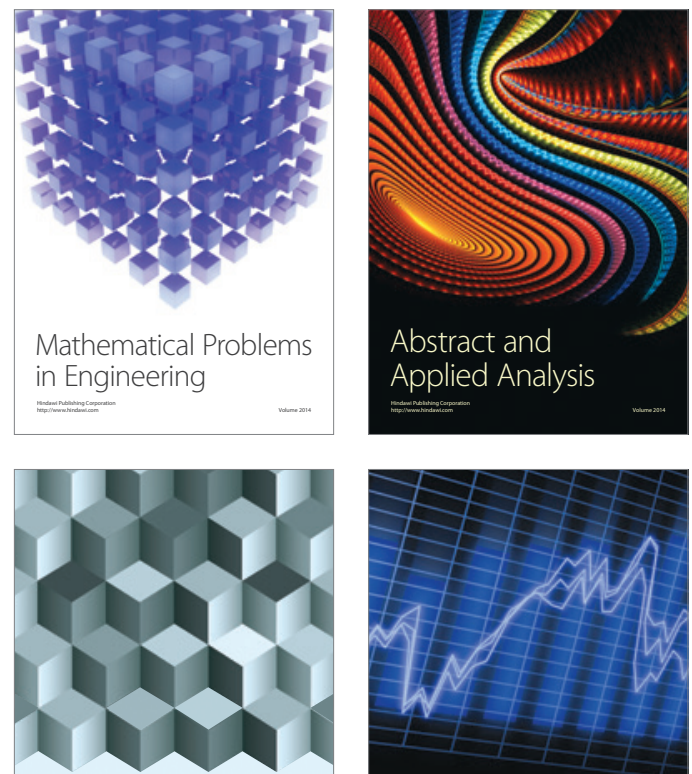

Journal of

Function Spaces

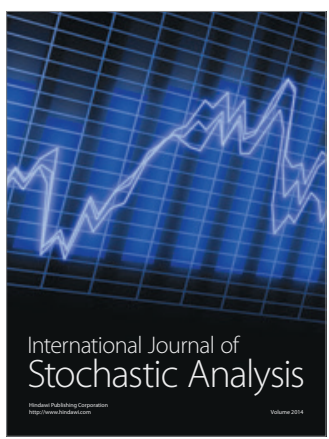

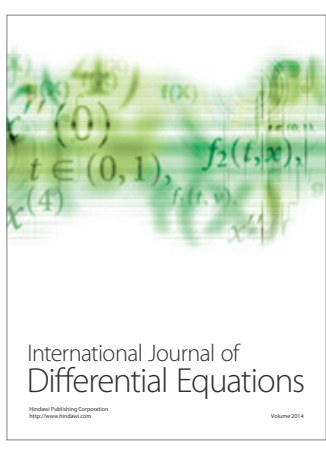
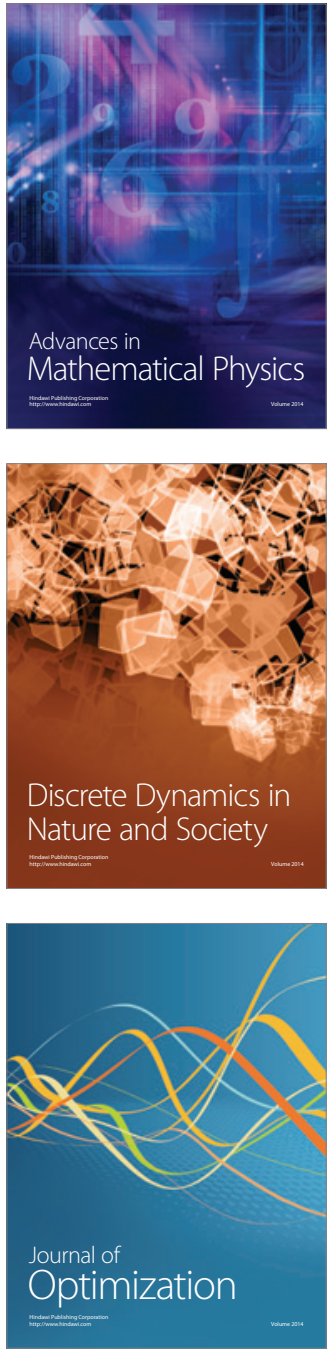